\section{Case Reports in Oncology}

\title{
Successful Perioperative and Surgical Treatment of a Rare Case of Extra-Gastrointestinal Stromal Tumor Arising in the Prostate Gland
}

\author{
Patrick Schöffski ${ }^{a} \quad$ Raf Sciot $^{b} \quad$ Maria Debiec-Rychter \\ Johan Van Ongeval ${ }^{d}$ André D'Hoore ${ }^{e} \quad$ Luc Merckx $^{f} \quad$ Steven Joniau $^{g}$ \\ aDepartment of General Medical Oncology, University Hospitals Leuven, Leuven Cancer

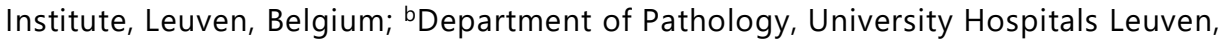 \\ Leuven Cancer Institute, Leuven, Belgium; 'Department of Human Genetics, University \\ Hospitals Leuven, Leuven Cancer Institute, Leuven, Belgium; dDepartment of \\ Gastroenterology, General Hospital Sint-Lucas, Ghent, Belgium; 'Department of \\ Abdominal Surgery, University Hospitals Leuven, Leuven Cancer Institute, \\ Leuven, Belgium; 'Department of Urology, General Hospital Sint-Lucas, Ghent, Belgium; \\ gDepartment of Urology, University Hospitals Leuven, Leuven Cancer Institute, \\ Leuven, Belgium
}

\section{Keywords}

Gastrointestinal stromal tumor · GIST · Prostate gland · KIT mutation · Interstitial prostatic cells $\cdot$ Interstitial cells of Cajal

\section{Abstract}

We report a very uncommon case of a primary, non-metastatic gastrointestinal stromal tumor (GIST) arising in the prostate gland in a 60-year-old patient. The morphology and immunohistochemical profile of the disease resembled GIST of gastrointestinal origin, and the molecular driver of this malignancy was a double mutation in exons 11 and 13 of the KIT gene. The tumor was proliferating slowly, did respond to neoadjuvant therapy with the KIT-inhibiting agent imatinib and was cured by radical, retro-pubic prostatectomy followed by adjuvant imatinib treatment. We postulate that primary GIST tumors of the prostate can arise from 
prostatic interstitial cells, which are the pacemakers of smooth muscle contractility in the gland, and possibly share a common precursor with typical GIST and the interstitial cells of Cajal in the gastrointestinal tract.

\section{Introduction}

Primary prostatic gastrointestinal stromal tumor (GIST) is an extremely rare entity with only a few cases reported in the world literature. While the vast majority of GIST originate from the gastrointestinal tract, GIST arising from extra-intestinal sites are very uncommon, and usually occur in anatomical localizations in close proximity to gastrointestinal structures, e.g. retroperitoneal, mesenteric or omental cases. In individual patients, rectal GIST can involve the prostate gland and mimic a primary prostate tumor. What we report here is a patient with a primary prostatic GIST without involvement of gastrointestinal structures.

\section{Case Report}

We describe the case of a 60-year-old male who presented in a regional hospital in 2009 with sporadic pollakisuria caused by a mass in the prostate gland of $12 \mathrm{~cm}$ in diameter. Figure 1 illustrates the radiological appearance of the tumour on pelvic computed tomography (CT). After initial watchful waiting until 2011 the patient underwent a biopsy of the prostate. Pathologic examination showed a mesenchymal tumor composed of spindle-shaped cells. On immunohistochemistry the tumor was positive for smooth muscle actin, CD34 and CD117 (KIT). Ki67 staining indicated a very low proliferation index of only $1 \%$. Mutational analysis of tumor tissue revealed a heterozygous, double mutation of KIT in exon 11 p.Gly565Arg (c. GGA>AGA) and in exon 13 p.Lys642Glu (c.1924A>G), most likely arising as a consequence of multiple mutagenic hits $[1,2]$.

The treating physicians decided to follow the patient without specific intervention, as he was almost asymptomatic and the disease appeared pretty stable over time. In 2015 he was transferred to the Department of General Medical Oncology at the University Hospitals in Leuven (Leuven, Belgium) for a second medical opinion and potential treatment.

The patient, a police officer who retired in 2014, had a general medical history of a sick sinus syndrome associated with paroxysmal arrhythmia, atrial flutter and tachycardia, thrombosis of the subclavian vein and a surgical history of vasectomy, implantation of a pacemaker and meniscus surgery. He had no relevant risk factors for malignancies and an unremarkable family history. He was in excellent condition when first presenting in our tertiary care clinic, was completely asymptomatic except for some mild dysuria/pollakisuria, and very reluctant to consider any active surgical treatment for the prostatic mass, given the fact that the tumor and the related symptoms had remained clinically stable over a period of 5 years.

The patient agreed though with staging procedures and a second transrectal biopsy to confirm the uncommon diagnosis. (18F)-fluorodeoxyglucose positron emission tomographycomputed tomography (PET CT) showed the presence of a metabolically active mass in the prostate gland, which had increased over time to $14 \mathrm{~cm}$ in diameter. The scan excluded the presence of another gastrointestinal or extra-gastrointestinal primary tumor or distant metastasis. The additional biopsy confirmed the working hypothesis of a low grade GIST arising in the prostate without major mitotic activity or spontaneous necrosis. Figure 2a shows the morphology of the tumor prior to any therapeutic intervention. Immunohistochemically the 
tissue stained positive for smooth muscle antigen, CD34, CD117 and Discovered on GIST 1 (DOG1) $[3,4]$. Figure $2 \mathrm{~b}$ illustrates the staining characteristics of the lesion.

Given the considerable size and documented slow progression of the primary tumor and the reluctance of the patient to undergo immediate primary surgery we strongly recommended to start systemic neoadjuvant treatment with imatinib, in analogy with our routine approach in patients with advanced rectal or esophageal GIST. With some delay the patient finally agreed in 01-2016 to start the tyrosine kinase inhibitor imatinib $400 \mathrm{mg}$ per day. His compliance was good and he tolerated the oral treatment very well. Adverse events were limited to episodic muscle cramps in the lower extremities and intermittent hypophosphatemia as classical side effects of imatinib. An early reassessment of the disease with PET CT within 4 weeks after the start of treatment showed an excellent partial metabolic response of the primary tumor and confirmed again the absence of distant metastases. Figures $3 \mathrm{~b}$ and $\mathrm{c}$ illustrate the radiological and metabolic response.

During further neoadjuvant treatment, his episodes of pollakisuria decreased in frequency and severity and he had excellent quality of life. In 04-2017 the primary tumor had shrunk to $9 \mathrm{~cm}$ in diameter on PET CT, with almost no residual (18F)-fluorodeoxyglucose uptake. In preparation of the resection of the tumor after neoadjuvant treatment a magnetic resonance tomography scan (MRT) of the pelvis was requested by the surgeons. This was performed in 11-2017 after replacing the patient's cardiac pacemaker by an MRT-compatible device. The MRT showed that the tumor was clearly confined to the prostate and had no anatomical relationship with the rectum or other intestinal structures.

In 12-2017 the patient underwent a radical retro-pubic prostatectomy performed by the urologist. Histology finally confirmed the diagnosis of a GIST of $7.3 \mathrm{~cm}$ in diameter after neoadjuvant treatment, located in the right lobe of the prostate, CD117 and DOG1 immunopositive. There was clear evidence of antitumoral effects of the preoperative therapy with imatinib but there was still presence of residual foci of vital tumor cells, which is a typical finding after neoadjuvant use of imatinib also in GIST arising in the gastrointestinal tract. Figure 2c shows the morphology of the tumor after imatinib treatment. As illustrated in Figure 4, mutational analysis was repeated, confirming the presence of the KIT exon 13 mutation c.1924A>G (p.Lys642Glu); the exon 11 mutation could not be detected anymore, which may be explained by differential effects of the kinase inhibitor on tumor cells with different driver mutations and higher sensitivity of the exon 11 mutation to imatinib.

After surgery the patient re-initiated treatment with imatinib at a dose of $400 \mathrm{mg}$ per day, which he is currently continuing until 01-2019 for a total perioperative treatment duration of 3 years, which is in line with current routine duration of adjuvant treatment in typical high risk gastrointestinal GIST. The patient is tolerating the postoperative treatment with imatinib even better than the neoadjuvant treatment, with no adverse events and no disease-specific complaints.

\section{Results}

At present, the patient is asymptomatic and tumor-free without any clinical or radiological evidence for residual disease. 


\section{Discussion/Conclusion}

GISTs are the most common mesenchymal malignancies in some geographic regions [5], and the most frequently encountered primary sarcoma arising in the gastrointestinal tract [6]. GIST tumors occurring outside of the gastrointestinal tract are extremely rare [7]. Primary GIST arising in the prostate have been reported in only a few cases [8-14].

The morphological, immunohistochemical and mutational features of the tumor described in this case report match the diagnosis of a GIST. A primary rectal origin of the disease could be ruled out both by extensive imaging studies and macroscopically during surgery. The clinical, radiological and metabolic sensitivity of the tumor to the KIT-directed tyrosine kinase inhibitor imatinib resembles the response pattern of classical gastrointestinal primary GISTs to this highly efficient and usually well-tolerated treatment. With the exception of the anatomical localization of the primary tumor, all features of this disease meet the diagnostic criteria of GIST.

How in theory can GIST arise in the prostate? The major function of the organ is to propel its secretions into the seminal fluids upon ejaculation, but the prostate in man and rodents also displays spontaneous electrical signals that trigger non-propelling contractile activity [15]. Specific prostatic interstitial cells are believed to be involved in processes involving contractility of smooth muscle fibers within the organ and to be responsible for the generation of the spontaneous tone in the prostate [16]. Electron microscopic studies in rodent prostates have established that numerous interstitial cells lay between the epithelium and muscle layer, and between and within the muscle bundles. These prostatic interstitial cells display surprising microstructural similarity with the interstitial cells of Cajal, the pacemaker cells in the gastrointestinal tract [17]. Furthermore, both the interstitial cells of Cajal in the bowel wall and the prostatic interstitial cells can be labelled with antibodies directed against the KIT receptor (CD117).

According to the literature, typical GISTs of the gastrointestinal tract are believed to share a common progenitor with the interstitial cells of Cajal, or even arise directly from these cells. Our working hypothesis, based on the case presented here, is that in rare instances GIST can also arise from prostatic interstitial cells, which in turn likely belong to the same superfamily as other interstitial pacemakers in the gastrointestinal and urogenital tract and possibly in other organ systems with contractility as a physiological function. Under mutagenic stress these cells can give rise to diseases with similar, if not identical phenotype, genotype and biological behavior as classical GIST of the intestine.

\section{Acknowledgement}

We want to acknowledge the contribution of involved radiologists and cardiothoracic surgeons in the care of this patient.

\section{Statement of Ethics}

The patient presented here provided written informed consent for the publication of this manuscript; the according consent form is part of his medical dossier. 


\section{Disclosure Statement}

The authors have no conflicts of interest to declare for this work.

\section{Funding Sources}

The authors did not receive any specific funding for this work.

\section{Author Contributions}

Patrick Schöffski performed the second opinion, coordinated the second biopsy, treated the patient with imatinib, obtained informed consent from the patient for publication of the article, wrote the manuscript and coordinated the contribution of other authors. He is also responsible for the follow-up of the patient.

Raf Sciot performed the histological and immunohistochemical assessment of the tumor and provided the according images and scientific input for the article.

Maria Debiec-Rychter did the mutational analysis prior to and after neoadjuvant therapy with imatinib and contributed to the writing of the article.

Johan Van Ongeval transferred the patient for the second medical opinion and contributed to the publication.

André D'Hoore excluded a gastrointestinal primary tumor in this patient and recommended to undergo prostatectomy.

Luc Merckx was responsible for the primary urological care of the patient at time of first diagnosis.

Steven Joniau performed the radical retro-pubic prostatectomy and contributed to the review of the manuscript.

\section{References}

1 Gasparotto D, Rossi S, Bearzi I, Doglioni C, Marzotto A, Hornick JL, et al. Multiple primary sporadic gastrointestinal stromal tumors in the adult: an underestimated entity. Clin Cancer Res. 2008 Sep;14(18):5715-21.

2 Rossi S, Gasparotto D, Toffolatti L, Pastrello C, Gallina G, Marzotto A, et al. Molecular and clinicopathologic characterization of gastrointestinal stromal tumors (GISTs) of small size. Am J Surg Pathol. 2010 Oct;34(10):1480-91.

3 Hemminger J, Iwenofu OH. Discovered on gastrointestinal stromal tumours 1 (DOG1) expression in nongastrointestinal stromal tumour (GIST) neoplasms. Histopathology. 2012 Aug;61(2):170-7.

4 Tang YL, Wong CF, Yap WM, Chuah KL. Discovered on gastrointestinal stromal tumours 1 (DOG1) expression in non-gastrointestinal stromal tumour (non-GIST) neoplasms. Histopathology. 2014 Nov;65(5):724-6.

5 Lurkin A, Ducimetière F, Vince DR, Decouvelaere AV, Cellier D, Gilly FN, et al. Epidemiological evaluation of concordance between initial diagnosis and central pathology review in a comprehensive and prospective series of sarcoma patients in the Rhone-Alpes region. BMC Cancer. 2010 Apr;10(1):150.

6 Miettinen M, Sarlomo-Rikala M, Lasota J. Gastrointestinal stromal tumours. Ann Chir Gynaecol. 1998;87(4):278-81.

7 Reith JD, Goldblum JR, Lyles RH, Weiss SW. Extragastrointestinal (soft tissue) stromal tumors: an analysis of 48 cases with emphasis on histologic predictors of outcome. Mod Pathol. 2000 May;13(5):577-85.

8 Zhang ZH, Feng GW, Liu ZF, Qiao L, Zhang T, Gao C, et al. A young man with primary prostatic extragastrointestinal stromal tumor: a rare case report and review of the literature. Int J Clin Exp Pathol. 2014 Mar;7(4):1764-70.

9 Liu S, Yu Q, Han W, Qi L, Zu X, Zeng F, et al. Primary gastrointestinal stromal tumor of the prostate: A case report and literature review. Oncol Lett. 2014 Jun;7(6):1925-9. 


\section{Case Reports in Oncology}

10 Van der Aa F, Sciot R, Blyweert W, Ost D, Van Poppel H, Van Oosterom A, et al. Gastrointestinal stromal tumor of the prostate. Urology. 2005 Feb;65(2):388.

11 Yinghao S, Bo Y, Xiaofeng G. Extragastrointestinal stromal tumor possibly originating from the prostate. Int ] Urol. 2007 Sep;14(9):869-71.

12 Lee $\mathrm{CH}$, Lin YH, Lin HY, Lee CM, Chu JS. Gastrointestinal stromal tumor of the prostate: a case report and literature review. Hum Pathol. 2006 Oct;37(10):1361-5.

13 Chang CF, Wen LW, Wu ST, Ho CL. An unusual case of primary prostatic extragastrointestinal stromal tumor. J Med Sci. 2016;36(2):78-80.

14 Reinke DA, Deisch JK, Reinke DD. Gastrointestinal stromal tumor with an unusual presentation as an enlarged prostate gland: a case report and review of the literature. J Gastrointest Oncol. 2016 Apr;7 Suppl 1:S71-4.

15 Lang RJ, Hashitani H. Role of prostatic interstitial cells in prostate motility. J Smooth Muscle Res. 2017;53(0):57-72.

16 Exintaris B, Klemm MF, Lang RJ. Spontaneous slow wave and contractile activity of the guinea pig prostate. ] Urol. 2002 Jul;168(1):315-22.

17 Huizinga JD, Faussone-Pellegrini MS. About the presence of interstitial cells of Cajal outside the musculature of the gastrointestinal tract. J Cell Mol Med. 2005 Apr-Jun;9(2):468-73.

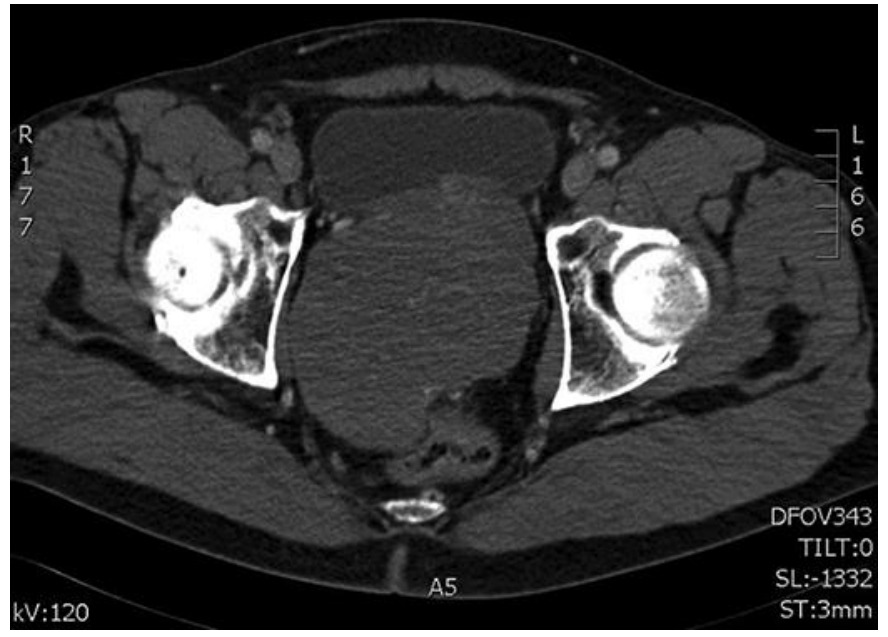

Fig. 1. Pelvic computed tomography (CT) scan performed in 10-2009 showing a large mass involving the prostate gland between the rectum and the urinary bladder. 


\section{Case Reports in Oncology}

Schöffski et al.: Successful Perioperative and Surgical Treatment of a Rare Case of Extra-Gastrointestinal Stromal Tumor Arising in the Prostate Gland
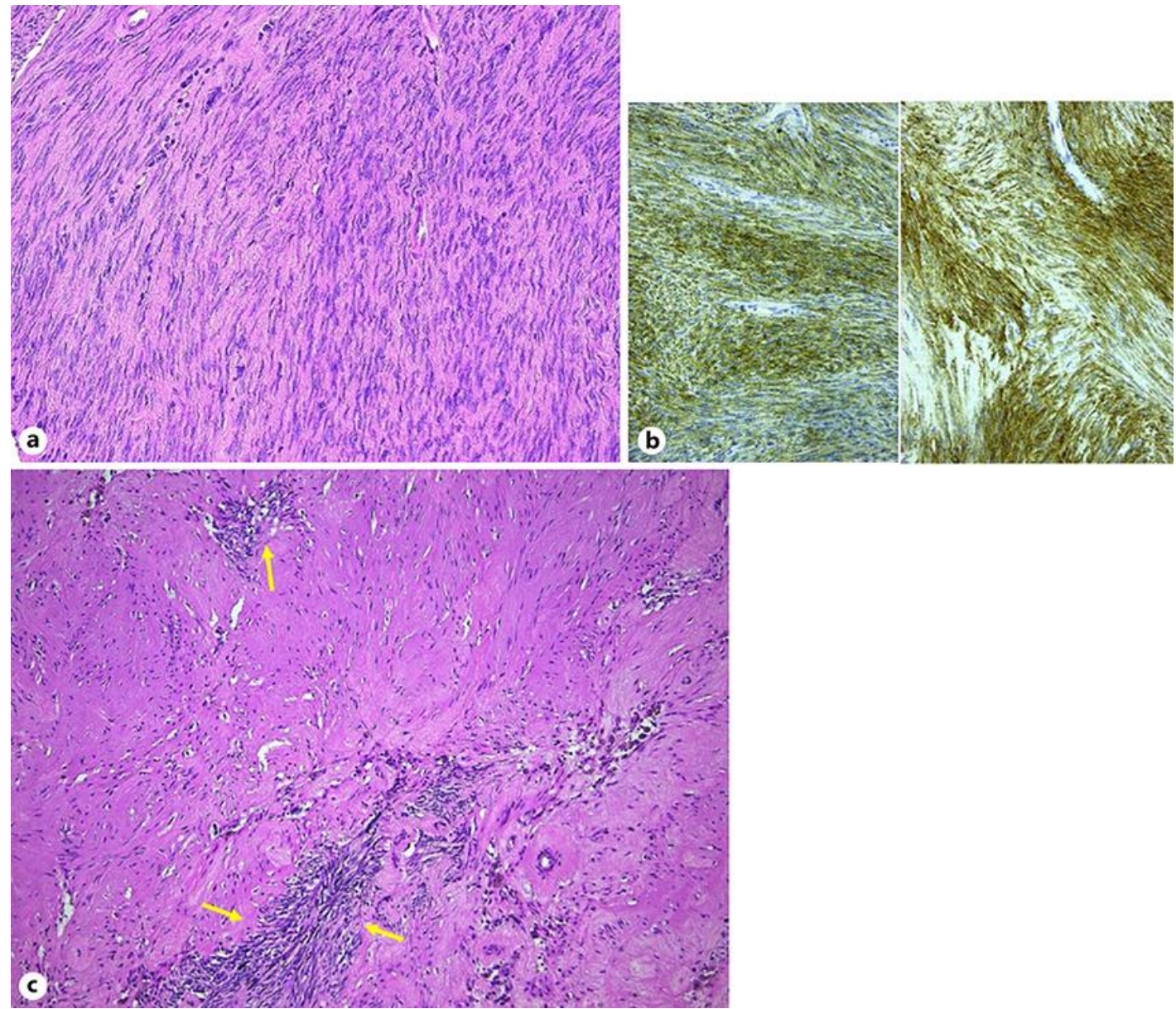

Fig. 2. a. Morphology of the primary tumor (H\&E stain, 400× magnification). Biopsy. The tumor consists of fascicles of non-atypical spindle cells with a buckled appearance. b. Immunohistochemical staining of the primary tumor (H\&E stain, 400× magnification). The tumor cells show a cytoplasmic granular expression of CD117 (left) and DOG1 (right). The vessel walls are negative. c. Histological response of the primary tumor to imatinib at time of prostatectomy (H\&E stain, 200× magnification). Areas of hyaline fibrosis are seen, with here and there cellular islands of residual tumor cells (arrows). 


\section{Case Reports in Oncology}
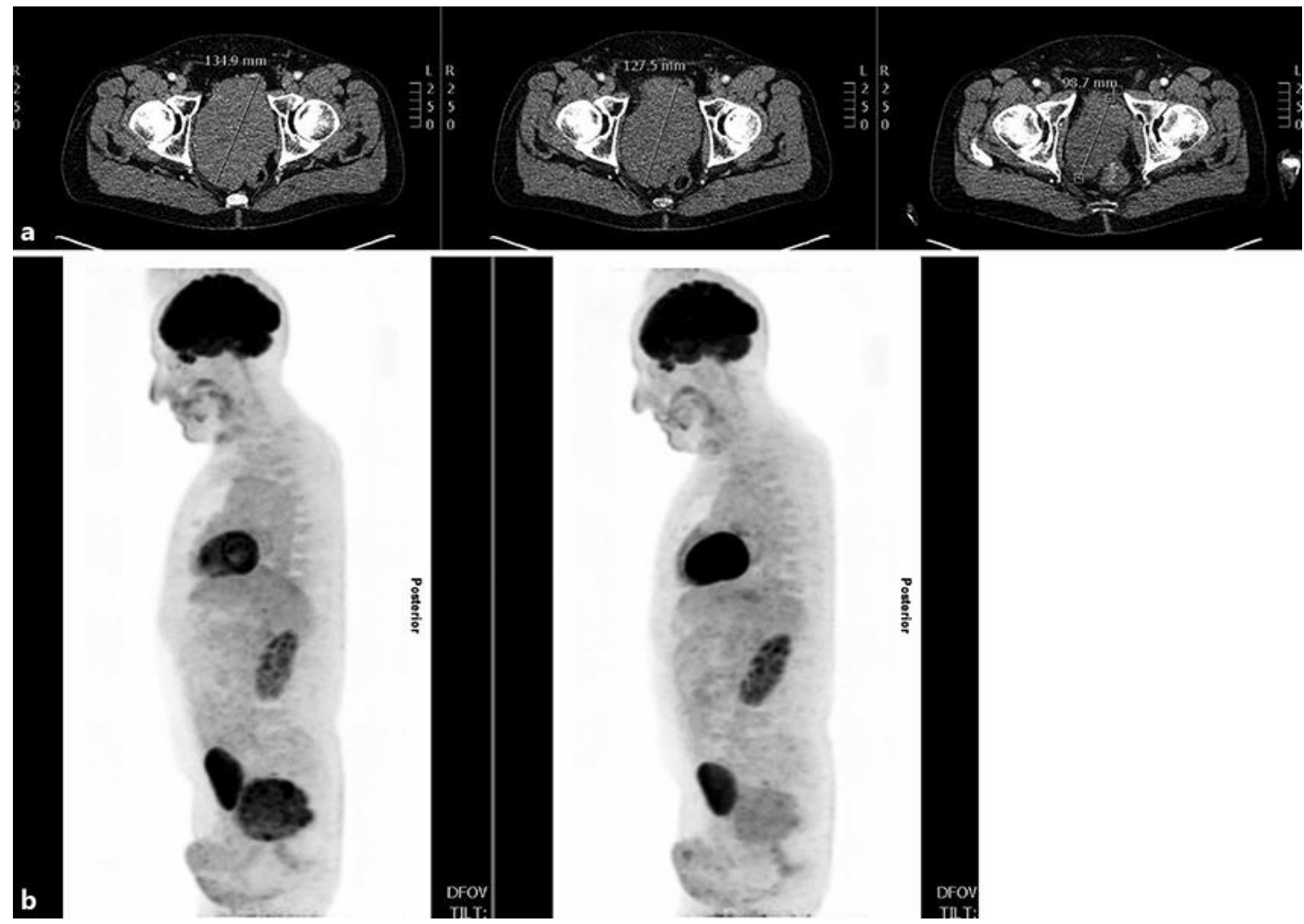

Schöffski et al.: Successful Perioperative and Surgical Treatment of a Rare Case of Extra-Gastrointestinal Stromal Tumor Arising in the Prostate Gland

Fig. 3. a. Computed tomography (CT) showing the volumetric response of the prostate tumor to imatinib after initiation of preoperative treatment with imatinib. Scans performed 12-2015, 02-2016 and 10-2016, imatinib $400 \mathrm{mg}$ p.o./day started 01-2016. b. (18F)-fluorodeoxyglucose positron emission tomographycomputed tomography showing the high glucose uptake of the retrovesical tumor prior to the start of imatinib treatment and metabolic response to the agent within 1 month after initiation of preoperative treatment. Scans performed 12-2015 and 02-2016, imatinib $400 \mathrm{mg}$ p.o./day started 01-2016. Note: the metabolic brain, heart, kidney and bladder signal is physiological. 
Schöffski et al.: Successful Perioperative and Surgical Treatment of a Rare Case of Extra-Gastrointestinal Stromal Tumor Arising in the Prostate Gland

A

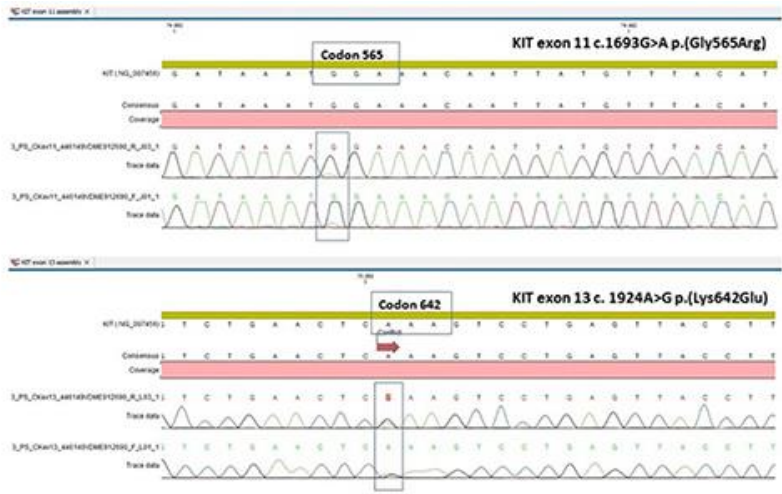

B
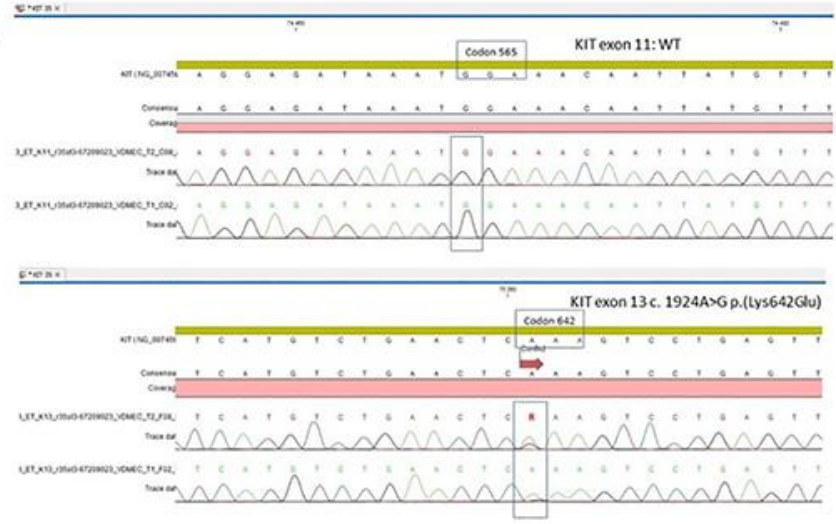

Fig. 4. Mutational analysis of the KIT gene in the primary tumor prior to neoadjuvant treatment revealing double KIT exon 11 and exon 13 mutations (A), while the tumor at time of resection carries only KIT exon 13 mutation (B). 